\title{
The successful surgical repair of a ruptured interventricular septum following a myocardial infarct in an 89 year old man
}

\author{
Richard M. Grocott-Mason ${ }^{1}$, Anthony Yates ${ }^{2}$ and Richard Wray \\ ${ }^{1}$ King's College Hospital, Denmark Hill, London, SE5 9RS, ${ }^{2}$ Guys Hospital, London and ${ }^{3}$ St Helen's \\ Hospital, Hastings, UK
}

\begin{abstract}
Summary: This case report describes the successful repair of an acquired ventricular septal defect following an anterior full thickness myocardial infarct in an 89 year old man. Four months after the infarct the patient was in severe congestive cardiac failure (NYHA Class IV), despite medical treatment, with signs of a ventricular septal defect. This was confirmed by echodoppler and cardiac catheterization. Surgical repair of the ventricular septal defect was performed. He made an uncomplicated recovery and two and a half years later is well and active. We believe he is the oldest patient who has had this operation successfully.
\end{abstract}

\section{Introduction}

Rupture of the interventricular septum occurs in approximately $1 \%$ of patients some 5 to 7 days after a transmural myocardial infarct. ${ }^{1}$ Surgical closure is the standard treatment. We report the successful surgical repair of a ruptured interventricular septum following an anterior myocardial infarct in an 89 year old man, leading to a marked improvement in his quality of life. We believe he is the oldest patient to have had such an operation successfully.

\section{Case report}

An 88 year old professional musician, was first seen in March 1987. He had a 2 week history of a chest pain and shortness of breath on minimal exertion.

On examination he was breathless on minimal exertion. Heart rate was $90 / \mathrm{min}$ regular. Blood pressure was $140 / 80 \mathrm{mmHg}$. Jugular venous pressure was raised at $4 \mathrm{~cm}$. At the apex and left sternal edge there was a grade $4 / 6$ pansystolic murmur and a soft third heart sound. Fine basal inspiratory crepitations were present. He had hepatomegaly but no peripheral oedema.

Electrocardiogram showed sinus rhythm with a recent extensive anteroseptal myocardial infarct. A

Correspondence: R.M. Grocott-Mason, M.R.C.P. Accepted: 10 December 1990 chest X-ray showed cardiomegaly with some pulmonary oedema. An echocardiograph the following month revealed a dilated left ventricle, which contracted reasonably well. The apical third of the septum was thin, akinetic and aneurysmal. There was a flow across the interventricular septum of $3 \mathrm{~m} . \mathrm{s}^{-1}$ detected on Doppler. He had some calcification of both the aortic and mitral valves with trivial mitral regurgitation.

He was treated with diuretics and digoxin but over the next 4 months progressively deteriorated. Despite increased medical therapy he steadily developed gross peripheral oedema, involving his legs and lower abdomen. He was in the New York Heart Association Functional Class IV. In July 1987 he was referred for consideration of surgical repair of his ventricular septal defect.

Right and left heart catheterization was performed. Left ventriculography confirmed an extensive apical infarct with a ventricular septal defect. The left anterior descending artery was occluded. Both the circumflex and the right coronary arteries were patent with no significant stenoses. Right atrial oxygen saturation was $50 \%$ and right ventricular oxygen saturation was $75 \%$. The pulmonary artery pressure was $85 / 20 \mathrm{mmHg}$ with a mean of $35 \mathrm{mmHg}$. Left ventricular end diastolic pressure was $20 \mathrm{mmHg}$.

The shunt size was calculated at 1.63:1. Following the catheterization he was treated with dobutamine and intravenous frusemide. His urea had increased to $15 \mathrm{mmol} / \mathrm{l}$. Four days later, at the age 
of 89 years and 17 days, he underwent surgical correction of his ventricular septal defect.

The operation was performed via a routine median sternotomy incision. There was a bifid apical left ventricular aneurysm, with an extensive old anterior left ventricular infarct. The aneurysm was excised leaving a defect in the left ventricle about $5 \mathrm{~cm}$ long. The ventricular septal defect was identified as a $1.5 \mathrm{~cm}$ diameter hole in the anterior part of the interventricular septum. This was closed with an elastic Dacron patch. The defect in the left ventricular wall was closed by direct suture. Immediately post-operatively his pulmonary artery pressure had reduced to $20 / 3 \mathrm{mmHg}$.

He made steady progress postoperatively. By the fifteenth postoperative day he was able to climb a flight of 10 steps independently.

His subsequent progress has been excellent. It is now almost 3 years since his operation and the patient is extremely active and well. Now, nearly 92, he drives to Spain, where he spends several months of the year, and his only medication is $20 \mathrm{mg}$ frusemide daily.

\section{Discussion}

The first successful surgical repair of a postinfarction ventricular septal defect was performed by Cooley et al in $1957 .^{2}$ The patient was a 49 year old male, operated on 11 weeks after an inferior myocardial infarct. After excellent initial progress the patient died after 45 days. Since this time surgery has come to be the standard treatment for this condition. With medical treatment less than $20 \%$ survive 1 month.

Once the surgical technique was established there was a lot of debate over the timing of the operation. In the early years the operation was electively delayed until 3-6 weeks following rupture of the septum. This produced good perioperative mortality rates of $10 \%$ and less. ${ }^{3,4}$ However, many patients either died before they had surgery or had serious complications. The recent trend has been towards early surgery. This has a higher perioperative mortality $(\sim 30 \%)$ but avoids many of the complications of prolonged circulatory collapse, such as renal failure, limb ischaemia secondary to intra-aortic balloon pumping, pneumonia and pressure sores. The most important prognostic indicator is the pre-operative haemodynamic state - hypotension, pulmonary oedema, renal failure and poor peripheral perfusion are all poor prognostic signs. ${ }^{4}$

Most of the statistics and reports on surgery for postinfarction ventricular septal defects are related to patients under 75. As cardiac surgery has advanced it has become clear that it is a suitable mode of treatment for the elderly. A recent review of over 100 cardiac operations on octogenarians has revealed a perioperative mortality of only $2 \%$ for elective coronary artery bypass grafting. ${ }^{5}$ The overall mortality for cardiac surgery in octogenarians was $16 \%$ (10\% for elective operations and $29 \%$ for urgent operations). Mortality was highest for patients needing preoperative inotropic support, intra-aortic balloon pumping and for those having a mitral valve replacement, either alone or in combination with revascularization or an aortic valve replacement. Only $29 \%$ of patients had an entirely uncomplicated post-operative course - the most frequent complication was atrial arrhythmia. Cerebral ischaemic events occurred in $20 \%$ of patients during their hospital stay. Over two thirds of these were transient. All patients with non-transient defects died. Of those patients who left hospital, $82 \%$ were alive at 2 years. The New York Heart Association functional class of patients was improved from a mean of 3.0 pre-operatively to 1.8 .

We are not aware of any reports in the literature of the surgical closure of a ventricular septal defect in any octogenarian patient. This case report shows that it is possible for very elderly patients, if appropriately selected, to survive major cardiac surgery and to derive enormous benefit. We feeb that patients who develop ventricular septal defects after acute myocardial infarcts should not be denied surgical treatment on the basis of age alone Whether elderly patients should also be considered for acute high risk intervention must be up to the individual surgeon and physician to consider the patient's wishes and their general condition. All the evidence suggests that pre-operative haemodynamic state is by far the most important prognostic determinant and that age is much less important. A thorough assessment of cerebrovascular circulation and the avoidance of nephrotoxic drugs is important. Surgery in this age group does not require long stays in hospital. Our patient stayed 10 days post-operatively at the regional centre and a further 1 weeks convalescence at the local hospital In the St Louis study the mean hospital stay was 14 days.

Our patient had selected himself into a relatively good prognostic group by surviving for more than 4 months with a ventricular septal defect acquired after an acute myocardial infarct. He was able to have a semi-elective operation which removed two mechanical causes for his cardiac failure. There are no data on the incidence of this particular problem in this age group and no other reports of surgical treatment. This report demonstrates how successful surgery can be in appropriately selected octogenarians. 


\section{References}

1. Fox, A., Glassman, E. \& Isom, O. Surgically remediable complications of myocardial infarction. Prog Cardiovasc Dis 1979, 21: $461-484$.

2. Cooley, D.A., Belmonte, B.A., Zeis, L.B. \& Schnur, S. Surgical repair of ruptured interventricular septum following acute myocardial infarction. Surgery 1957, 41: 930-937.

3. Daggett, W.M., Buckley, M.J., Akins, C.W. et al. Improved results of surgical management of postinfarction ventricular septal rupture. Ann Surg 1982, 196: 269-277.

4. Norell, M.S., Gershlick, A.H., Pillai, R. et al. Ventricular septal rupture complicating myocardial infarction: is earlier surgery justified? Eur Heart $J$ 1987, 8: 1281-1286.

5. Naunheim, K.S., Dean, P.A., Fiore, A.C. et al. Cardiac surgery in the octogenarian. Eur J Cardio-thorac Surg 1990, 4: $130-135$. 